\title{
Dynamics of Intracluster Gas and Bulk Motions in Clusters
}

\author{
RENATO DUPKE
}

Department of Astronomy, University of Michigan, Ann Arbor, Michigan, USA

\begin{abstract}
In this paper I briefly discuss progress in recent lines of research on the internal dynamics of clusters of galaxies that have been made possible thanks to improvements in current $X$-ray spectrometers that enable them to perform detailed spatially resolved spectroscopy. In particular, I focus on the study of bulk motion in intracluster gas and the nature of features called cold fronts.
\end{abstract}

KEYWORDS: dynamics; intracluster gas; bulk motions

Clusters of galaxies are the largest gravitationally bound systems in the universe. As such, they are interesting not just for their intrinsic nature, structure, and evolution, but also for their use as tools in cosmology. For example, if they represent a fair sample of the early universe their baryonic mass fraction $f_{b}$ value should be equal to $\Omega_{b} / \Omega_{m}$, where $\Omega_{b}$ and $\Omega_{m}$ are the baryon and total mass densities of the universe, respectively, normalized to the critical density. ${ }^{1}$ The baryon fraction can be combined with the value of $\Omega_{b}$ as predicted from light element abundances through big bang nucleosynthesis, ${ }^{2}$ to provide constraints on $\Omega_{m}{ }^{3,4}$ (see FIGURE 1). Since galaxies amount to only a small fraction of the baryonic mass in clusters, the precision with which the baryon fraction in clusters is estimated depends heavily on how well we can determine the intracluster gas mass, which is the dominant baryonic component and makes up typically $15-20 \%$ of the total cluster mass.

The intracluster gas is very hot $\left(10^{7}-10^{8} \mathrm{~K}\right)$, highly ionized, and emits mostly in $\mathrm{X}$-rays through a combination of Bremstrahlung and line emission (see FigurE 2). The X-ray emitting mass is calculated through a combination of X-ray surface brightness profile and spectral fittings. Typically, the surface brightness (proportional to $n^{2} T^{1 / 2}$ for a hydrogen plasma) profile is fitted with some deprojected King-like profile proportional to $\left(1+\left(r / r_{0}\right)^{2}\right)^{-3 \beta+0.5}$, where $\beta$ is the ratio of specific energies of galaxies and gas $\left(\mu m_{p} \sigma_{\text {gal }}^{2} / k T\right)$. To determine the total mass, the X-ray method usually relies on the assumption of hydrostatic equilibrium (HE), which gives the mass enclosed within radius $r$ as $M(r) \propto-r T(\Delta r T+\Delta r \rho)$, where $T$ and $\rho$ are the gas temperature and density, respectively, and $\Delta r X$ is the logarithmic derivative $(d \ln X / d \ln r)$.

The degree of applicability of the above mentioned working assumptions is an important current issue, since it is now known that clusters are not spherically symmetric virialized systems as was previously thought, but instead they are dynamically active and have significant substructures, as shown by recent X-ray and optical

Address for correspondence: Renato Dupke, Department of Astronomy, University of Michigan, Ann Arbor, MI 48109, USA.

rdupke@umich.edu

Ann. N.Y. Acad. Sci. 1045: 260-275 (2005). (C2005 New York Academy of Sciences. doi: 10.1196/annals.1350.022 


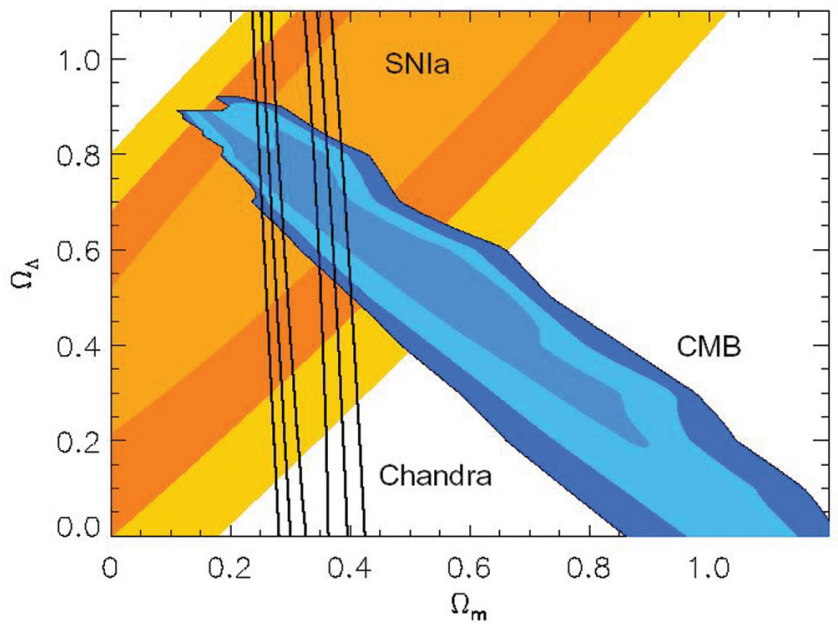

FIGURE 1. Constraints on $\Omega_{m}$ as derived from clusters of galaxies, overlaid on 1, 2, and $3 \sigma$ contour plots from studies of the anisotropies of the CMB and from SN Ia. (Reproduced from Ref. 4 with permission.)

studies. Shocks, cold fronts, buoyant bubbles, temperature anisotropies, and velocity gradients are often found in these systems. Detailed knowledge of the dynamical phenomenology of clusters is important, not just to understand their origin and evolution, but also to use them as cosmological tools. The primary process of formation and growth of galaxy clusters is the merging of collapsed subsystems. This formation

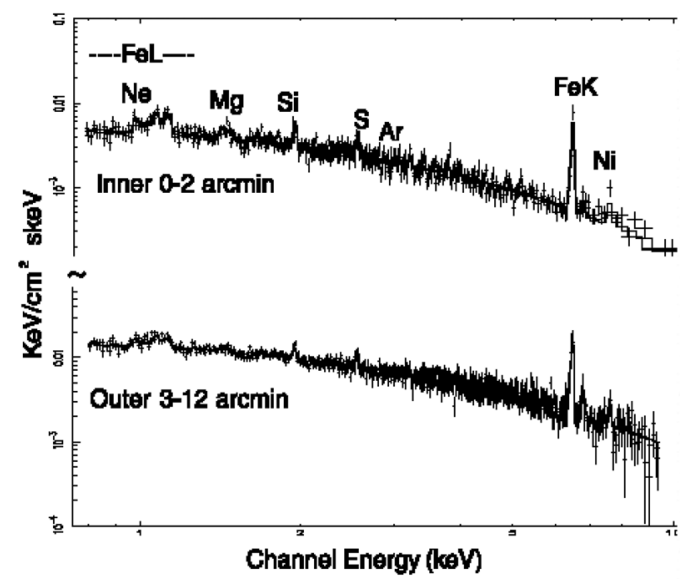

FIGURE 2. Illustration of X-ray spectra with ASCA GIS. Spectra of inner 2' (top) and outer ( $\left.3^{\prime}\right)$ (bottom) of Abell 496. The main spectral lines and line complexes are indicated. 


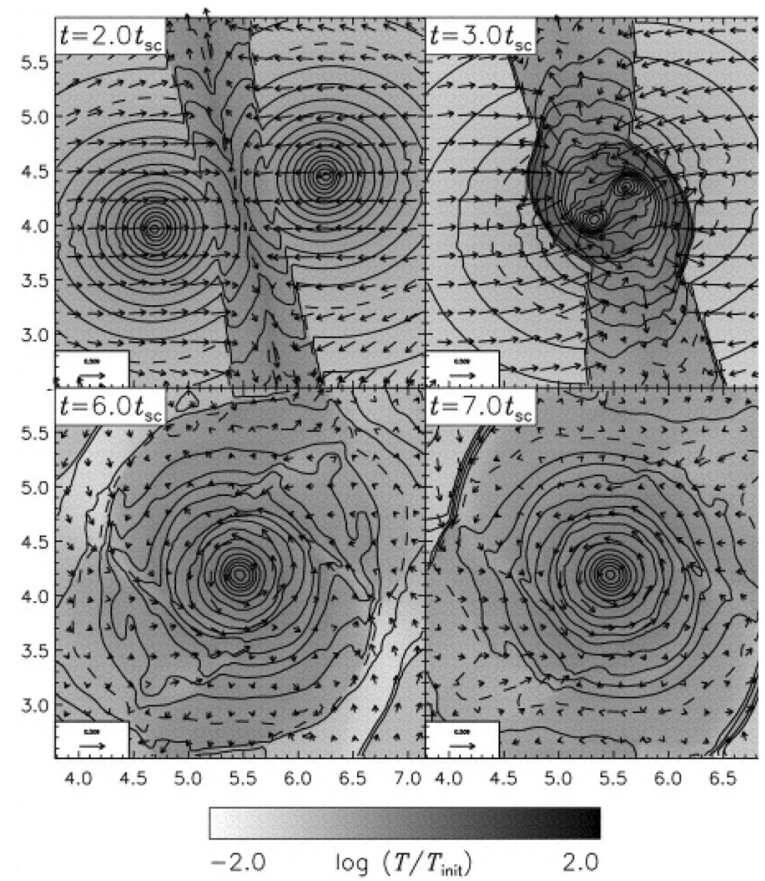

FIGURE 3. Off-center collision of clusters where initial impact parameter is about 10 core radii. Shading indicates the logarithm of the temperature, $t_{\mathrm{sc}}$ is the crossing time (Gyr). Velocity arrows are drawn for every eighth cell, with the fiducial arrow at the corner of each plot representing $v \sim 2,000 \mathrm{~km} / \mathrm{sec}$. (Reproduced from Ref. 5 with permission.)

process is within the framework of the so-called bottom-up hierarchical scenario for the origin of clusters, where larger structures build up from small to large scales, via merger/accretion of smaller size systems. It is predicted that these mergers will be more frequent along larger filamentary structures that characterize the "cosmic web".

The most notable expected dynamical process of the ICM is clusters off-center merging (for a recent review see Sarazin ${ }^{6}$ ). This process is believed to create strong temperature, density, gas velocity, and also chemical substructures. 5,7 The link between temperature substructure and the merger stage is often made by comparison with hydrodynamic simulations. There is currently an enormous variety of cluster formation/merger simulations in the literature, ${ }^{5,8-19}$ see also <vce.physics.lsa. umich.edu>. These simulations provide, among other quantities, temperature and surface brightness (or gas density) maps that can be compared with observations. In particular, off-center merging imparts angular momentum to the intracluster gas, which, according to recent numerical and hydro simulations, can last several $\mathrm{Gyr}^{5,16,20-22}$ (see FIGURE 3). Measurements of bulk gas flows are crucial to track the evolutionary stage of clusters breaking the usual degeneracies that appear when comparing only at projected temperatures and densities to the simulations. 


\section{BULK MOTIONS}

To date most of evidence of gas bulk flows in clusters in indirect (e.g., A2218, ${ }^{23}$ A2142, A3667, and 1E0657-56 24-26). We have recently shown that it is possible to measure bulk gas velocities directly with currently available spectrometers. ${ }^{27,28} \mathrm{We}$ determine gas velocities by measuring redshift differences in the emission lines in the $\mathrm{X}$-ray spectra from cluster gas in different directions (regions). A line centroid can be measured with a precision $\Delta V \approx 127 \Gamma_{\mathrm{ev}} E_{\mathrm{kev}}^{-1} N^{-1 / 2} \mathrm{~km} \cdot \mathrm{sec}^{-1}$, where $N$ is the number of photons in the line and $\Gamma_{\mathrm{ev}}$ is the FWHM of the line, or if the line is narrower than the instrumental width, it is the FWHM of the instrument, and $E_{\mathrm{kev}}$ is the line energy. Currently, the energy resolution (FWHM) of ACIS-S3 on-board Chandra varies from $3 \%$ to $3.6 \%$ at $5.9 \mathrm{keV}$ depending on the chip row, $<$ http://space.mit.edu/ACIS/acismemo10.html >. This would, in principle, allow us to measure a line centroid $\left(\mathrm{FeK}_{\alpha}\right)$ to a precision of $500-1,000 \mathrm{~km} / \mathrm{sec}$ with only a few hundred line photons. However, the ability to measure velocities with this precision is not merely a matter of collecting a sufficient number of photons. The main difficulty one faces in determining bulk velocities to know precisely the conversion between pulse-height and energy (gain), how it changes across the detectors and also over time. The gain fluctuations are typically on the same order or larger than the velocities one is trying to measure, so that taking the gain fluctuations into account properly is crucial to determine reliable velocity gradients.

The advanced satellite for cosmology and astrophysics (ASCA) was the first satellite to have the minimum conditions for velocity studies, high energy resolution, recorded gain variations across the detectors, and reasonably good gain stability. The first cluster where ICM velocity gradients were found was Perseus, where we discovered, using ASCA, evidence for large-scale gas bulk motions of more than $1,500 \mathrm{~km} / \mathrm{sec}$ at the $90 \%$ confidence level, consistent with either ICM circulation or streaming motions due to a large merger event. ${ }^{27}$ In Centaurus, where excellent data from the ASCA solid state spectrometers (SIS) were available, we found a significant (greater than $99 \%$ confidence level) velocity gradient at small scales (less than $5^{\prime}$ ), ${ }^{28}$ see Figure $4 \mathrm{~A}$.

Since the main uncertainties of measuring velocities using X-ray spectroscopy is knowing well the intrinsic variations of the gain, it is extremely important: (1) to corroborate the measurements using other instruments, since it is unlikely the gain systematics will be the same, and (2) to develop strategies to minimize the dependence on gain. We used (1) and (2) for the Centaurus clusters by using the Chandra satellite to perform two observations of the cluster, one centered in the region of maximum velocity and the other centered in the region of minimum velocity using the same chip position, ${ }^{29}$ thus avoiding having to deal with intrachip gain variations. Chandra off-center pointings allowed us not just to confirm but also to improve ASCA measurements. The maximum velocity difference found was $(3.4 \pm 1.0) \times 10^{3} \mathrm{~km} / \mathrm{sec}$ and showed that the velocity distribution is more consistent with the presence of "eddies" than with "bulk rotation". Furthermore, the characteristic energy associated with this eddie is about $10^{61}(\mu / 0.6)\left(n_{\text {gas }} / 0.01 \mathrm{~cm}^{-3}\right)\left(V_{\text {circ }} / 1200 \mathrm{~km} / \mathrm{sec}\right)^{2}\left(L / 4^{\prime}\right)^{3} \mathrm{ergs}$ ! The presence of multiple eddies in clusters may provide non-thermal pressure support that can significantly affect mass and energetics estimates.

The analysis of multiple pointings with Chandra and XMM specifically tailored to minimize gain variations can benefit from a prior knowledge of the configuration 

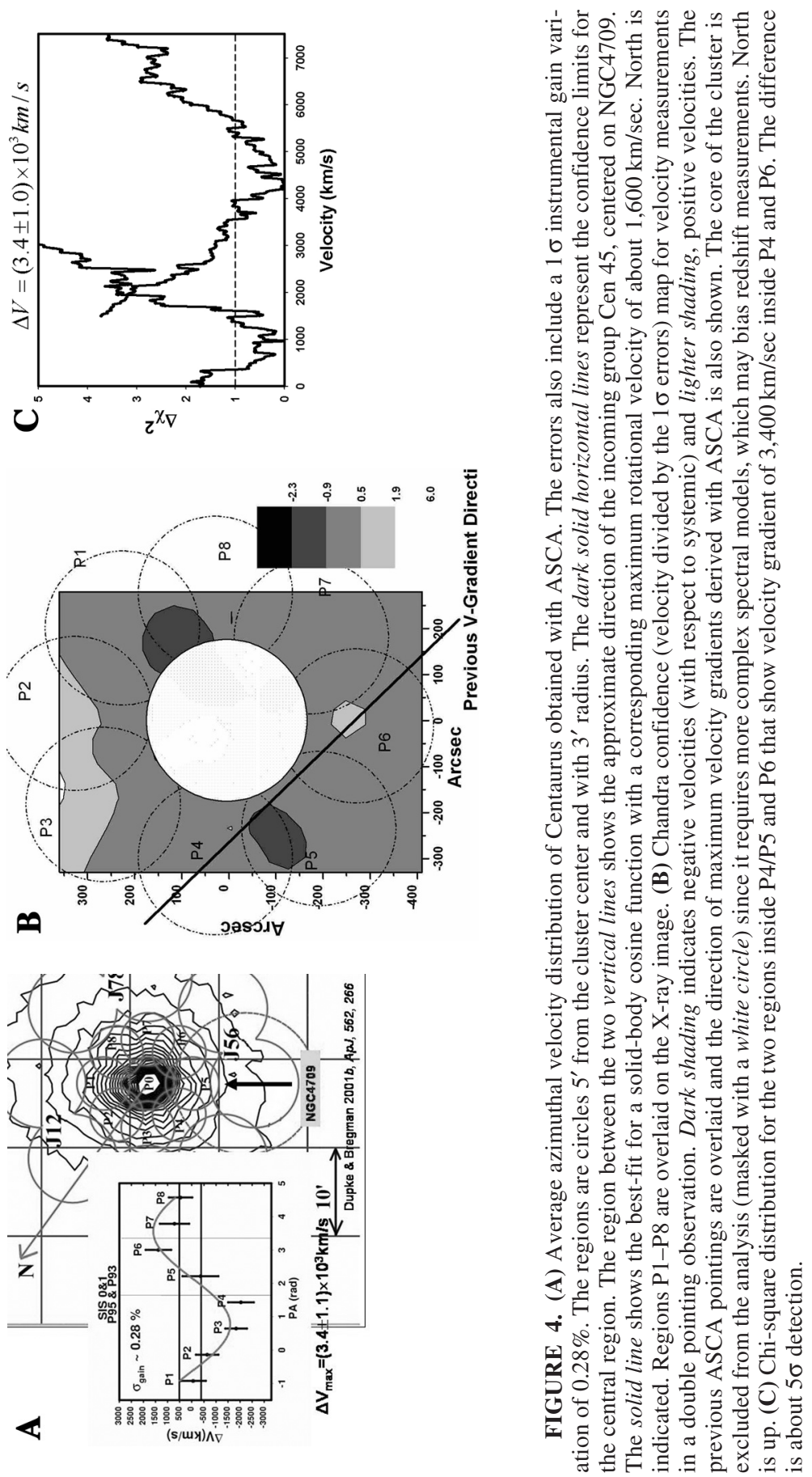
of the velocity gradients and ASCA can provide it for clusters with very high bulk velocities. After the initial success in detecting velocity gradients in Perseus and Centaurus we carried out a systematic search in the ASCA archives for clusters with velocity substructure. We have recently finished this search ${ }^{30}$ and we found three more clusters where we detected internal velocity gradients greater than $1,000 \mathrm{~km} / \mathrm{sec}$ (at $90 \%$ confidence level). Their internal velocity distribution (normalized by the errors) are shown in FIGURE 5.

The cluster found with the highest and most reliable velocity gradient is Abell 576. Abell 576 is a richness class 1 cluster with low central gas temperatures and average metal abundances. It has an optical redshift of 0.0389. ASCA velocity analysis of this cluster found a significant velocity gradient of more than $4,000 \mathrm{~km} / \mathrm{sec}^{30}$ with respect to the inner Eastern regions. The high velocity gradient found in Abell 576 adds to the body of indirect evidence to the presence of high dynamical activity in this cluster. Rines et al. ${ }^{31}$ determined the mass profile of A576 using the infall pattern in velocity space for more than 1,000 galaxies in a radius of $4 \mathrm{~h}^{-1} \cdot \mathrm{Mpc}$ from the center of the cluster. They found that the mass of the central Mpc was more than twice that found from X-ray measurements, suggesting that non-thermal pressure support may be biasing the X-ray derived mass. Their result is also in agreement with previous mass estimates of Mohr et al., ${ }^{32}$ who found a high velocity tail, separated by about $3,000 \mathrm{~km} / \mathrm{sec}$ from the mean of the cluster. Kempner et al. ${ }^{33}$ analyzed the Chandra observations of the core of this cluster and found sharp edges corresponding to jumps in gas density and pressure roughly in the $\mathrm{N}-\mathrm{S}$ direction and suggested that the core substructures are caused by a current merger with tangential core velocities of about $750 \mathrm{~km} \cdot \mathrm{sec}^{-1}$.

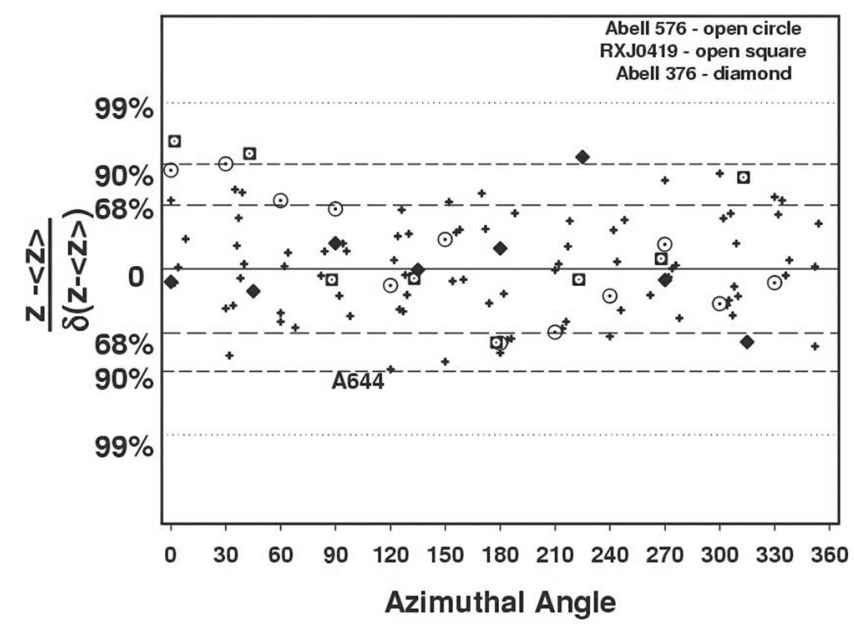

FIGURE 5. Error weighted distribution of relative velocities: we show all subregions, for all clusters in the ASCA sample. The 68\% (long dashed lines), 90\% (short dashed lines), and $99 \%$ (dotted lines) distribution boundaries are also shown. The clusters with the highest significant velocity structures are indicated by different symbols: Abell 576 (open circles), RX J0419+0225 (open squares), and Abell 376 (diamonds). 

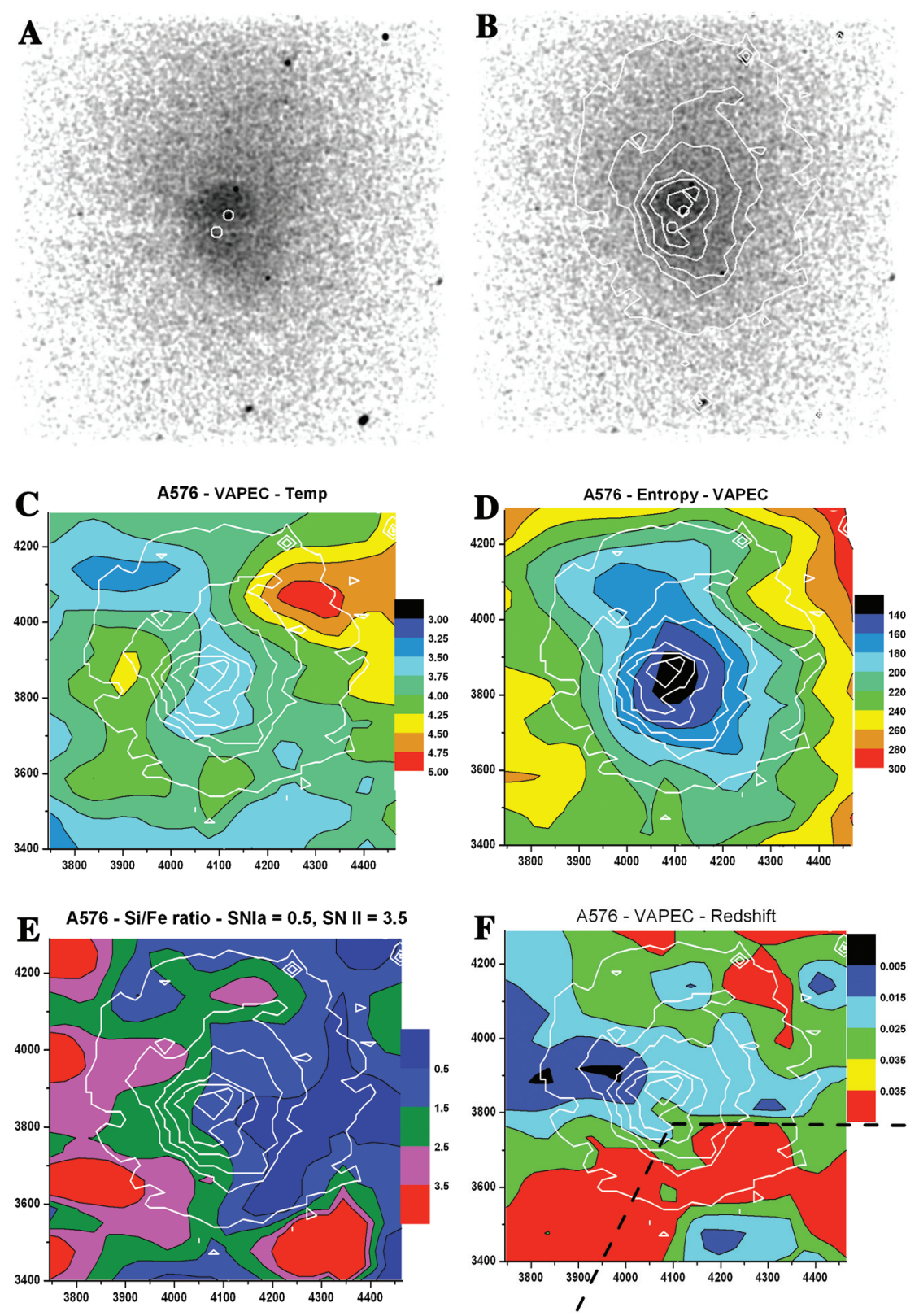

FIGURE 6. 
In Figure 6A we show a Chandra X-ray image of A576. Two features stand out: a surface brightness arm from the center to the S-SE forming a near triangular wedge pointing $\mathrm{E}$ and also wakes (or fingers) can be seen in the NW region. These are expected in supersonic galaxy motions. ${ }^{34}$ FIGURE $6 \mathrm{~B}$ shows the same as FIGURE $6 \mathrm{~A}$ but with surface brightness contours overlaid. FIGURE 6C-F show colored contour maps of the distribution of the interesting parameters obtained through spectral fittings using an absorbed VAPEC thermal emission model.

The best fit results for individual points in the map are a result of adaptive smoothing with more than 5,000 cnts/cell and a minimum smoothing radii of 70 pix $\left(35^{\prime \prime}\right)$. The color steps were chosen so as to approximate the $1 \sigma$ errors. FIGURE $6 \mathrm{C}-\mathrm{F}$ show the distribution of gas temperatures $(\mathrm{keV})$, entropy $\left(\mathrm{cm}^{2} \cdot \mathrm{K}\right), \mathrm{Si} / \mathrm{Fe}$ ratio, and redshifts. These plots suggest a merger incoming from the NW direction. The X-ray arm is associated with pressure enhancement toward the East with the projected temperature rising by $30 \%$ and with marginal indications of a temperature reduction after the shock front (toward the Eastern CCD border). There are two D galaxies near the core of the cluster and they are plotted as circles in FIGURE 6A. Both galaxies are offset from the X-ray isophotal center. The extended low velocity region from the center toward East shows that the merger is also happening near the line of sight going toward the observer. The velocity gradient is significant at the $90 \%$ confidence level and shows a difference of $(6 \pm 3.2) \times 10^{3} \mathrm{~km} / \mathrm{sec}$, in agreement a previous ASCA velocity analysis of similar regions. ${ }^{30}$

The region of high velocities found with ASCA is the sector indicated by the dashed lines in FIGURE 6F. An XMM pointing of similar (but smaller due to CCD gaps) regions also detected a velocity gradient of $(2.8 \pm 1.7) \times 10^{3} \mathrm{~km} / \mathrm{sec}^{34} \mathrm{The}$ precision of velocity measurements in that observation was limited by the loss of exposure due to extended flare periods. Although each of the cited individual measurements is not statistically conclusive, per se, the fact that different instruments with different systematics are showing velocity differences in the same regions suggests that the presence of velocity gradients is robust.

We show the projected distribution of the Si/Fe ratios in FigurE 6E. The importance of this ratio is that it allows us discriminate of SN type enrichment, serving as a fingerprint for the gas enrichment history. This is because SNe Ia and II produce different amounts (yields) of different elements. Therefore, we can use elemental abundance ratios to determine the contribution from SN Ia and II to the X-ray emitting plasma. ${ }^{35}$ For example, classical SN Ia models predict that the ratio of $\mathrm{O}$ to $\mathrm{Fe}$ abundances (by number normalized to Solar) should be about 0.04 , whereas SN II models predict the same ratio to be about 3.8. Thus, if the measurement of the $\mathrm{O} / \mathrm{Fe}$ ratio in the IGM is 0.5 , it would mean that the SN Ia iron mass fraction is $88 \%$, and the rest (about 12\%) would have been produced by SN II. The enrichment history depends on the previous internal characteristics of the merging systems, such as amount of gas stripped by galaxies, SN Ia winds from the central galaxy, strength of protogalactic SN II dominated winds, and so forth. If the edges mentioned above were all generated by some internal phenomena, not connected to a merger, we should see no asymmetry in the distribution of $\mathrm{Si} / \mathrm{Fe}$ ratio. However, what we see is exactly the opposite. The cluster core sits on the border of a significant separation of media with different enrichment histories. Toward the $\mathrm{E}$ the ratio goes from 1.5 near the X-ray arm to 3.5 at $3^{\prime}$ from the center corresponding to $0-65 \%$ SN Ia 
contamination. Toward the $\mathrm{W}$ the ratio decreases from 1.0 near the cluster core to 0 at $1.5^{\prime}$ away. This corresponds to $85-100 \%$ SN Ia contamination. The difference is significant at more than $90 \%$ confidence level and provides direct evidence that the X-ray arm is a surface discontinuity between two different media. The SN Ia enriched "bullet" is trying to push its way through a SN II enriched medium.

The projected temperatures derived for this cluster seem unusually low for such a violent merger. However, two factors must be considered. The first is that the thermalization of the kinetic component could be altered by a pre-existent velocity gradient (gas circulation) from a previous merger. The second is that we are determining projected quantities and since the merging is happening near the line of sight, the effects of projection become more important and we are likely to underestimate the derived gas temperatures. By looking at the temperature map, we can see that the shocked region (E-SE) extends to the South and has a peak directly E of the sharp edge. Selecting the region with the maximum temperature and using a 2-T model we obtain a best-fit hot component $T_{\text {high }}=6.5_{-1.2}^{+2.8} \mathrm{keV}$. The cool component is $T_{\text {low }} \sim 2 \mathrm{keV}$.

Such high bulk velocities lead to an underestimate of the X-ray derived total mass under the assumption of hydrostatic equilibrium, since there is an extra non-thermal pressure not taken into account. This underestimate of the X-ray derived mass when compared to independent mass estimators can be used as a rough indicator of velocity gradients, if systematics are taken into account. One popular independent method to determine cluster masses is gravitational lensing. Typically, lens modeling methods rely on the detection of as many lensed background galaxies as possible, and the inversion of the lensing effect of the cluster to recover its mass distribution. Lensing techniques are used to determine the projected mass and are sensitive to uncertainties in the three-dimensional configuration, such as elongations on the line-of-sight.
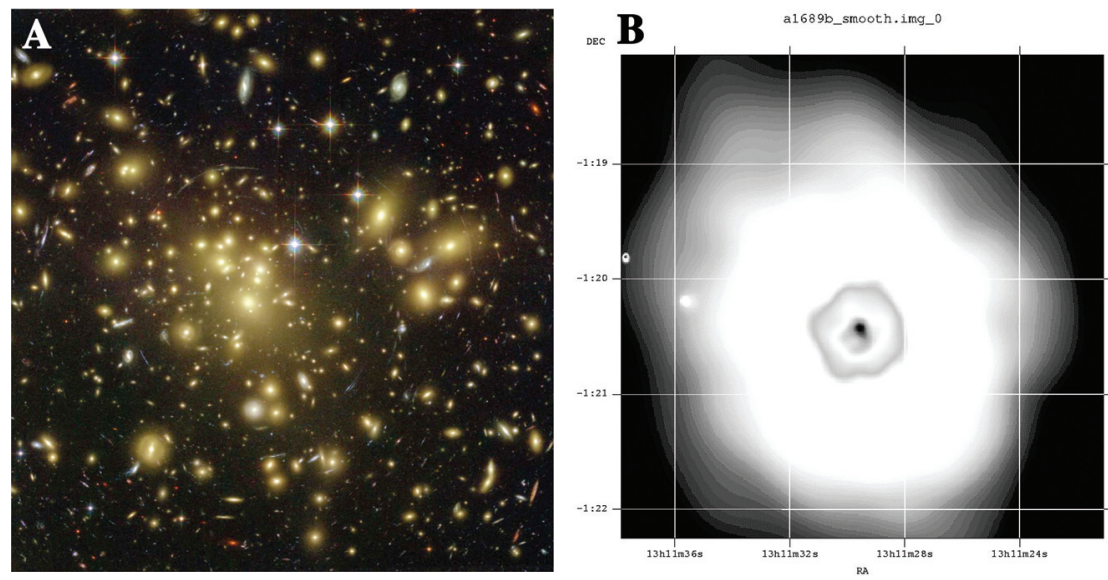

FIGURE 7. (A) ACS-WFC image of the lensing cluster Abell $1689(z \sim 0.18)$. The image is 200 " on one side. (Reproduced from Ref. 36 with permission.) (B) Chandra image $(10 \mathrm{ksec})$ of A1689, roughly on the same scale. 
$\mathrm{X}$-ray and lensing masses do not always agree; often the mass determined through $\mathrm{X}$-ray analyses fall short when compared to those determined through lensing (hereafter X-ray-lens discrepancy). Interestingly, one of the most persistent discrepancies happens to be in the most powerful lens known, A1689 ${ }^{38,39}$ (see FIGURE 7) Given its optimal physical characteristics, A1689 is a natural laboratory for testing the consistency of different techniques for mass measurements and their limitations. MiraldaEscude and Babul $^{40}$ were the first to notice a discrepancy by a factor of two in the cluster masses determined through lensing and X-ray. The authors suggested several explanations for the lower X-ray mass, among them multitemperature gas and nonthermal pressure support, such as intracluster gas bulk motions in the center. Allen ${ }^{41}$ was able to reproduce the mass derived from lensing in most of the clusters of his sample, using a combination of ASCA and ROSAT HRI observations and elaborated techniques to compensate for the overestimation of the $\mathrm{X}$-ray core radius sizes. This, however, still could not reconcile the discrepancy in A1689. Andersson and Madejski ${ }^{39}$ analyzed A1689 with XMM and confirmed the factor of two discrepancy. However, they performed a velocity analysis, similar to those described above and found a strong velocity gradient of about $5,000 \mathrm{~km} / \mathrm{sec}$ near the clusters center, indicating that the cluster is in the initial stages of a major merging in the line-of-sight.

The frequency of high velocity gradients is higher than expected theoretically, given the small number of clusters observed. Pawl, Evrard, and Dupke ${ }^{42}$ have used the virtual cluster exploratory database of mock clusters from a $\Lambda$ CDM cosmological simulation to analyze the frequency and magnitude of velocity gradients in the intracluster gas. They found that the likelihood to find velocity gradients greater than $\Delta v$ decreases as $(\Delta v)^{-4}$, and that only $6 \%$ of clusters showed velocity gradients larger than half of the sound speed (see FIGURE 8). Given the current observation difficulties involved in the analysis of intracluster velocities it is likely that the sample of clusters analyzed for velocity gradients so far is biased toward the richest, brightest,

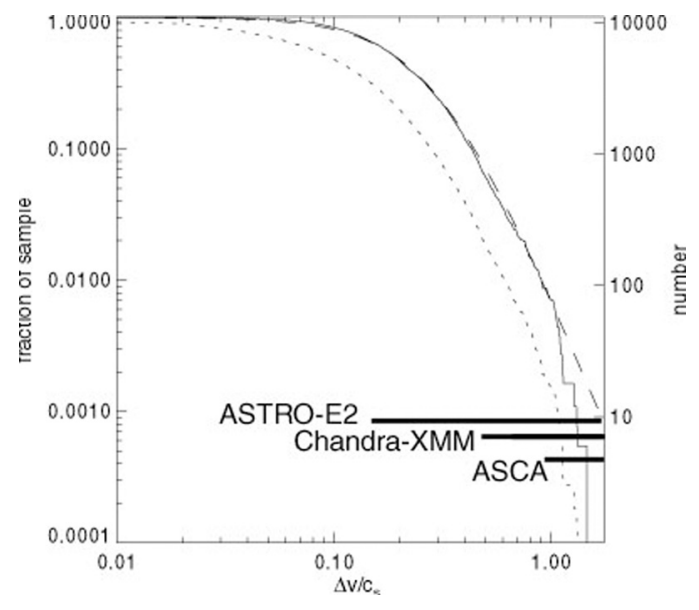

FIGURE 8. Cumulative likelihood of velocity gradients normalized by the ICM sound speed for the maximum velocity difference in a cluster (solid line) and for all regions analyzed in all clusters (dotted line). (Reproduced from Ref. 41 with permission.) 
and most likely to be undergoing strong dynamical processes. In the next few years the study of velocity gradients in intracluster gas is likely to become a major source of information about the nature of cluster formation and the systematics involved in using them as cosmological probes. Larger archival searches with Chandra and XMM will be able to provide better observation constraints to the high velocity tail of the expected frequency of velocity gradients. ASTRO-E2 impressive energy resolution (sensitive to velocity uncertainties of about $100 \mathrm{~km} / \mathrm{sec}$ ) will provide unprecedented bulk velocity measurements as well as turbulent motions through extrathermal line broadening. ${ }^{43}$ However, ASTRO-E2 performance will benefit greatly from new Chandra and XMM observations specifically tailored for velocity studies. This is because ASTRO-E2 lifetime is short (two years) and the X-ray calorimeter has a small field of view $\left(2.9^{\prime} \times 2.9^{\prime}\right)$ and collecting area and, without prior knowledge of the overall velocity distribution, it will need a large number of observations to map a single cluster.

\section{Cold Fronts}

Another indication of dynamic activity in clusters is given by the frequent presence of cold fronts. Cold fronts are sharp surface brightness discontinuities characterized by a jump in gas temperature, accompanied by a decline in gas density, such that the gas pressure remains continuous across the front. These characteristics make cold fronts different from bow shocks and the origin for their formation remains unclear. The initial explanation for cold fronts is associated to subsonic (transonic) motions of accreted substructures, ${ }^{24,25,44}$ such as gas clumps or small galaxy groups with suppressed thermal conduction. In FIGURE 9A we show schematics for this model from Markevitch et al. ${ }^{24}$ The top image shows the previous merger epoch. The bottom one shows the stage where cold fronts are seen. Shock fronts have propagated to the cluster outer regions, and the dense core keeps moving through the shocked gas without mixing.

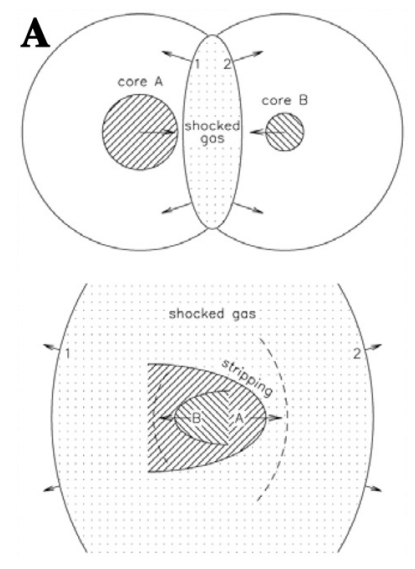

B

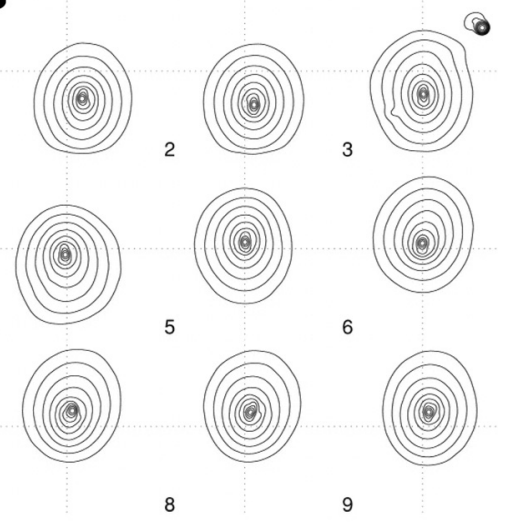

FIGURE 9. 
Models that suggest merger core remnant, such as that described above (external models), are theoretically justified ${ }^{45,46}$ and hold relatively well for clusters that have clear signs of merging, such as $1 \mathrm{E} 0657-56^{26}$ and A3667. ${ }^{44}$ However, they do not work well for the multiple cold fronts discovered in clusters that do not show other strong merging signs, such as $\mathrm{A} 496^{7}$ and A1795. ${ }^{25}$ This inconsistency has led to the creation of alternative models for cold fronts that involve internal mechanisms, such as oscillation of the $\mathrm{cD}$ plus low entropy gas around the bottom of the potential well $7,47,48$ and gravitational field oscillation due to scattering of smaller systems. ${ }^{49,50}$ We show a plot from Tittley and Henriksen ${ }^{49}$ (FIG. 9B) that illustrates the creation of multiple cold fronts due to the passage and deflection of another system, for illustration. The incoming group approaches from the left in the first two frames (each frame is $50 \mathrm{Myr}$ apart and is $200 \mathrm{kpc}$ in size) and is scattered downwards continuing out of view. In frame 3 closest approach happens (about $100 \mathrm{kpc}$ ). The resulting compression of the isodensity contours due to the field oscillation (dark matter and baryonic matter become detached) happens several frames after the deflection, when the deflected group is far away from the core. External models such as this have the advantage of allowing for the formation of multiple coexisting cold fronts.

As pointed out by Dupke and White, ${ }^{7}$ internal and external models for cold front formation can be discriminated through the analysis of SN Ia/II contamination (or relative metal enrichment) across the front. If the cold front generation mechanism is external we should expect that the front was also be accompanied by a discontinuity of metal enrichment histories. The gas enrichment history can be determined through the measurement of an ensemble of metal abundance ratios as explained in the previous section. It is unlikely that both metal abundances and abundance ratios would conspire to fake a smooth distribution during merging. Also, if the accreted cluster is the core of a "non-cooling flow" cluster its metal abundance would be that of the average value for clusters $(0.3$ solar $)$ making it easily distinguishable from the metal enriched central region of the main cluster. If, on the other hand, the cold front is generated by internal mechanisms one would not expect strong chemical gradients at the cold front, except maybe for those associated with the cluster itself, such as radial abundance gradients.

The analysis of heavy element distribution in cold fronts is a powerful tool to understand their nature and currently has to be done with (or in addition to) Chandra, given its combination of very high angular and spectral resolutions. Dupke and White ${ }^{7,47}$ performed a chemical analysis of the cold front in A496. This is an apparently well behaved nearby bright cluster at a redshift of 0.033 . It does not show any obvious signs of merging. At least two sharp edges are seen in the X-ray surface brightness and projected images (see FIGURE 10 A). Both edges can also be seen easily in the temperature maps (FIG. 10C). The North edge is characterized by a jump in temperature that can be as high as 1.6 times, accompanied by a density reduction of about 1.8 , with a continuous radial decline in pressure $(n k T)$. The gas temperature continues to grow radially in that direction achieving a maximum of about $6 \mathrm{keV}$ within the region analyzed. The overall characteristics are typical of cold fronts. The temperature distribution in the southern edge is anisotropic. The temperature jumps by about 1.3 times to at least $4.0 \mathrm{keV}$ toward the S-SE and the density drops by about 1.7 times over the same spatial scale, consistent with other cold fronts. To the SW 

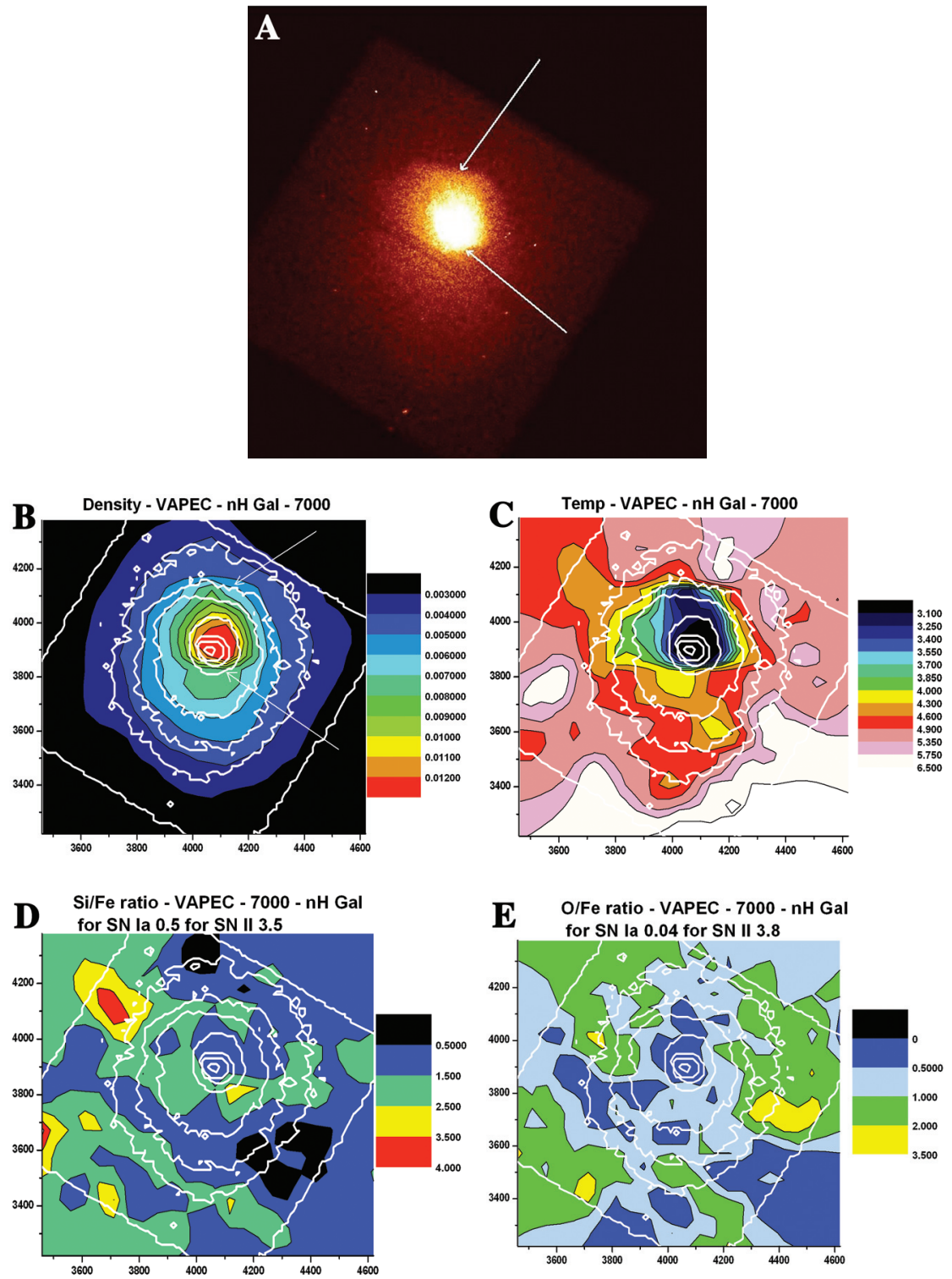

FIGURE 10. Contour maps of the distribution of the interesting parameters obtained through spectral fittings using an absorbed VAPEC thermal emission model. The best fit results for individual points in the map are a result of adaptive smoothing with more than $7,000 \mathrm{cnts} / \mathrm{cell}$ and a minimum smoothing radii of 20 pix $\left(10^{\prime \prime}\right)$. The color steps were chosen in a way as to approximate the $1 \sigma$ errors. B-D show the distribution of gas density $\left(\mathrm{cm}^{-3}\right)$, temperatures $(\mathrm{keV}), \mathrm{Si} / \mathrm{Fe}, \mathrm{O} / \mathrm{Fe}$, and redshifts. The outermost square contour shows the CCD border and measurements outside this should be ignored. 


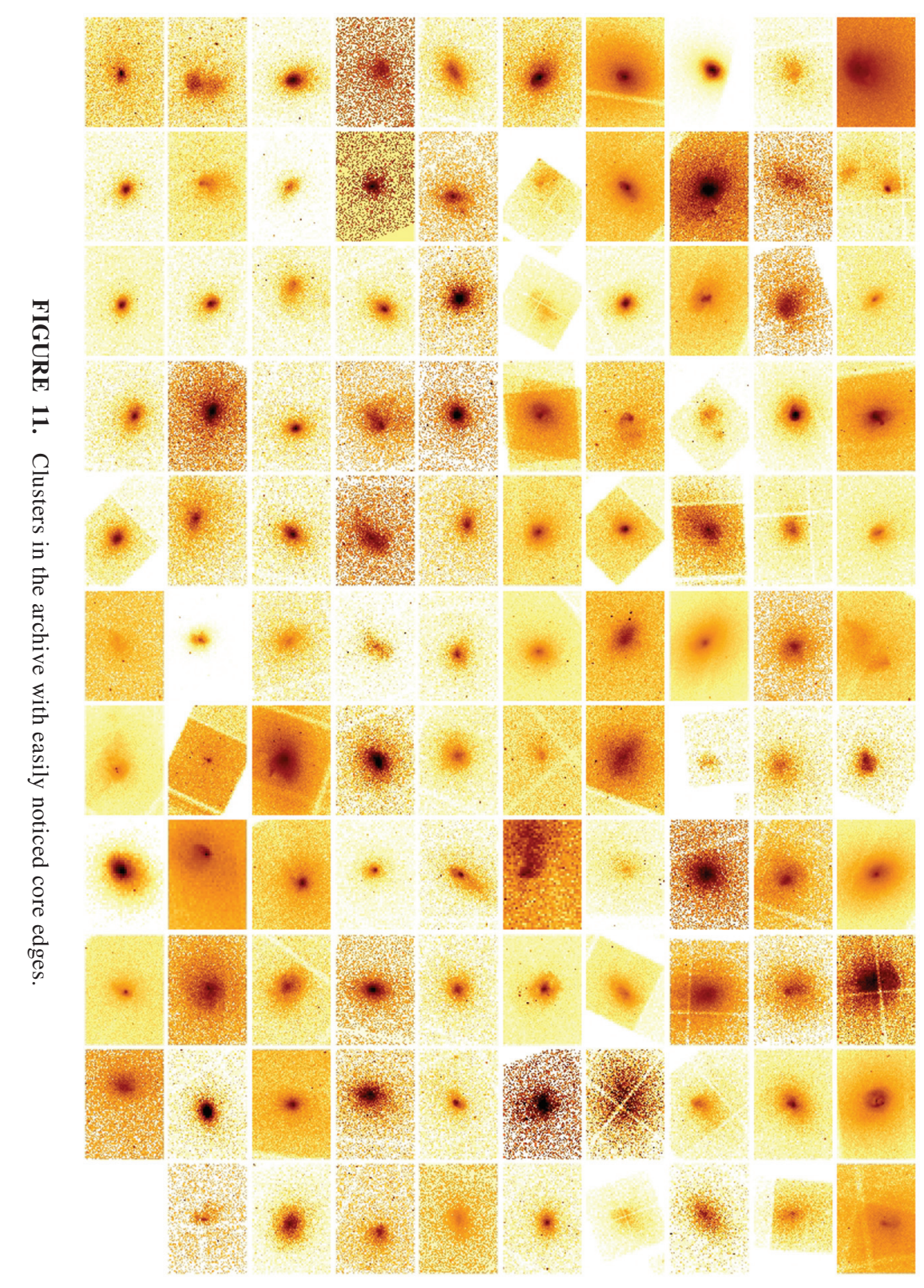


the temperature has a stronger gradient, reaching $4.6 \mathrm{keV}$ (a near 1.6 enhancement) for the same density decline, creating an isobaric tail.

The distribution of $\mathrm{Fe}, \mathrm{Si}$, and $\mathrm{O}$ abundances showed overall radial gradients, but there were no clear discontinuities related to the cold fronts. The only discontinuities across the cold fronts were consistent with general radial gradients. Both $\mathrm{O} / \mathrm{Fe}$ and $\mathrm{Si} / \mathrm{Fe}$ are consistent with a central dominance of SN Ia ejecta (FIG. 10D and E). Within the errors, there was no major change in abundance ratio distribution across the sharp boundaries of the cold front. This is compatible with an internal mechanism to generate cold fronts, for example, the $\mathrm{cD}$ is oscillating around the cluster potential well. In this model, the $\mathrm{cD}$ would be dragging/smearing $\mathrm{SN}$ Ia iron enriched cold gas within the spatial oscillation length (about $80 \mathrm{kpc}$ ), which defines the distance to cold front.

The velocity map in the regions where it can be well constrained (near the core) suggests that the region of the high temperature tail ( $\mathrm{S}-\mathrm{SW})$ sits on a lower-thanaverage velocity (by more than $300 \mathrm{~km} / \mathrm{sec}$ at $1 \sigma$ ). This suggests that the temperature tail represents stripped material with residual velocities along the line-of-sight towards the observer and also North (up) ( $Z-Y$ directions). The moving core, in this scenario, turned around $(\mathrm{N})$ and is currently moving away from the observer.

There are now a large number of clusters observed with Chandra and a significant fraction of them show signs of sharp edges near the core (see FIGURE 11). If most of these edges are real cold fronts it seems unlikely that merging of subclumps is the only way to make them. This is because of their high frequency (near 50\%) and the lack of intermediate stages in the accretion process (e.g., before core passage). However, all the mechanisms proposed involve some dynamic activity, either with mergers, scattering of subclumps or sloshing of cold gas and/or cD in the bottom of the potential well. Combined with temperature, density, pressure, and entropy distriubtions, a systematic SN Type enrichment study of cold fronts will provide fundamental clues as to their origin and impact on the dynamics of the intracluster gas.

\section{ACKNOWLEDGMENT}

I thank Steve Gottesman for the excellent work in organizing the conference on which this volume is based and for allowing Henry's friends and collaborators to honor him one more time. I also thank Joel Bregman, John Arabadjis, Narciso Benitez, and Nestor Mirabal for helpful discussions. This work was partially supported by NASA Grants 603-4162X, NNG046H856, AR4-5013X, and G04-5145X.

\section{REFERENCES}

1. White, S.D., J.F. Navarro, A.E. Evrard \& C.S. Frenk. 1993. Nature 366: 429.

2. Schramm, D. \& M. Turner. 1998. Rev. Mod. Phys. 70: 303.

3. Bahcall, N.A., J.P. Ostriker, S. Perlmutter \& P.J. Steinhardt. 1999. Science 284: 148.

4. Allen, S., R. Schmidt \& A. Fabian. 2002. Mon. Not. Roy. Astr. Soc. 334: L11.

5. RICKER, P.M. 1998. Astrophys. J. 496: 670.

6. SARAZIN, C. 2004. astro-ph/0406181.

7. Dupke, R. \& R. White. 2003. Astrophys. J. 583: 13. 
8. Evrard, A.E. 1990. Astrophys. J. 363: 349.

9. KATZ, N. \& S.D.M. White. 1993. Astrophys. J. 412: 455.

10. Roettiger, K., J. Burns \& C. Loken. 1993. Astrophys. J. 407: 53.

11. Roettiger, K., J. Burns \& C. LoKen. 1996. Astrophys. J. 473: 651.

12. Schindler, S. \& E. Muller. 1993. Astron. Astrophys. 272: 137.

13. Pearce, F.R., P.A. Thomas \& H.M.P. Couchman. 1994. Mon. Not. Roy. Astr. Soc. 268: 953.

14. Takizawa, M. \& S. Mineshige. 1998. Astrophys. J. 499: 82.

15. TAKIZAWA, M. 1999. Astrophys. J. 520: 514.

16. TAKIZAWA, M. 2000. Astrophys. J. 532: 183.

17. Navarro, J.F., C.S. FrenK \& S.D.M. White. 1995. Mon. Not. Roy. Astr. Soc., 275: 720.

18. Evrard, A.E., C.A. Metzler \& J.F. Navarro. 1996. Astrophys. J. 469: 494.

19. Roettiger, K., C. LoKen \& J.O. Burns. 1997. Astrophys. J. 109: 307.

20. Gómez, P.L., C. Loken, K. Roettiger \& J.O. Burns. 2002. Astrophys. J. 569: 122.

21. Motl, P.M., J.O. Burns, C. Loken, et al. 2004. Astrophys. J. 606: 635.

22. Roettiger, K. \& Flores. 2000. Astrophys. J. 538: 92.

23. Machacek, M., M.W. Bautz, C. Canizares \& G.P. Garmire. 2002. Astrophys. J. 567: 188.

24. Markevitch, M., et al. 2000. Astrophys. J. 541: 542.

25. Markevitch, M., A. Vikhlinin \& P. Mazzotta. 2001. Astrophys. J. 562: L153.

26. Markevitch, M., A.H. GonZalez, L. David, et al. 2002. Astrophys. J. 567: 27.

27. DupKe, R.A. \& J.N. BREgMAN. 2001. Astrophys. J. 547: 705.

28. Dupke, R.A. \& J.N. Bregman. 2001. Astrophys. J. 562: 266.

29. Dupke, R.A. \& J.N. Bregman. 2005. Astrophys. J. Submitted.

30. Dupke, R.A. \& J.N. Bregman. 2005. Astrophys. J. Submitted.

31. Rines, K., M.J. Geller, A. Diaferio, et al. 2000. Astrophys. J. 120: 2338.

32. Mohr, J., M.J. Geller, D.G. Fabricant, et al. 1996. Astrophys. J. 470: 724.

33. Kempner, J. \& L. DAvid. 2004. Astrophys. J. 607: 220.

34. Stevens, I., D. Acreman \& T. Ponman. 1999. Mon. Not. Roy. Astr. Soc. 310: 663.

35. Dupke, R.A., N. Mirabal \& J.N. Bregman. 2005. In preparation.

36. Loewenstein, M. \& R. Mushotzky. 1996. Astrophys. J. 466: 695.

37. Broadhurst, T., et al. 2005. Astrophys. J. 621: 53.

38. XuE, S. \& X. WU. 2002. Astrophys. J. 576: 152.

39. Andersson, K. \& G. MAdejSKi. 2004. Astrophys. J. 607: 190.

40. Miralda-Escude, J. \& A. Babul. 1995. Astrophys. J. 449: 18.

41. Allen, S. 1998. Mon. Not. Roy. Astr. Soc. 296: 392.

42. Pawl, A., A. Evrard \& R. Dupke. 2005. Astrophys. J. Submitted.

43. Sunyaev, R.A., M.L. Norman \& G.L. Bryan. 2003. Astron. Lett. 29: 783.

44. Vikhlinin, A., M. Markevitch \& S.S. Murray. 2001. Astrophys. J. 551: 160.

45. BialeK, J. \& A. Evrard. 2002. Astrophys. J. 578: 9.

46. Nagai, D. \& A. Kravtsov. 2003. Astrophys. J. 587: 514.

47. Lufkin, E., S.A. Balbus \& J.F. Hawley. 1995. Astrophys. J. 446: 529.

48. Dupke, R. \& R. White. 2005. In preparation.

49. Tittley, E. \& M. Henriksen. 2005. Astrophys. J. 618: 227.

50. Ascasibar, Y. \& M. MarkeVITCH. 2005. In preparation. 